Published in final edited form as:

J Case Rep Med. 2014 ; 3: . doi:10.4303/jcrm/235859.

\title{
Tracheobronchial amyloidosis: A case report and review of the literature
}

Andrew C. Birkeland, M.D. ${ }^{1}$, Jonathan B. McHugh, M.D. ${ }^{2}$, and Matthew E. Spector, M.D. ${ }^{1}$ ${ }^{1}$ Department of Otolaryngology - Head and Neck Surgery, University of Michigan Health System, Ann Arbor, Michigan

${ }^{2}$ Department of Pathology, University of Michigan Health System, Ann Arbor, Michigan

\begin{abstract}
Objectives-This is a case report on tracheobronchial amyloidosis. This disease may provide a diagnostic challenge for otolaryngologists due to its rarity and relative lack of literature. Our case highlights presentation and workup of this disease.

Methods-The index patient's workup included clinical exam, CT of the neck and chest, direct laryngoscopy, bronchoscopy and biopsy. Additional reports of tracheobronchial amyloidosis were identified in a PubMed database search.

Results-Our index patient presented with dyspnea and hoarseness. Clinic laryngoscopy showed a tracheal mass. Radiology demonstrated an irregular tracheal soft tissue lesion. The patient underwent biopsy, and pathology was consistent with amyloidosis. He had no systemic signs of amyloidosis. He underwent local resection to improve his airway diameter, with no complications.

Conclusions-Tracheobronchial amyloidosis should be kept in an otolaryngologist's differential diagnosis for patients with nonspecific upper airway symptomatology and radiographic lesions in the trachea or bronchi.
\end{abstract}

\section{Keywords}

Amyloidosis; tracheobronchial; dyspnea

\section{Introduction}

Amyloidosis is a well-studied systemic disease consisting of systemic protein deposition in extracellular tissue. Most commonly, the heart, liver, kidneys and skin are affected. Isolated tracheobronchial amyloidosis, however, is a rare disorder with amyloid deposits limited specifically to tracheal and bronchial tissue. This disease may provide a unique diagnostic challenge for otolaryngologists due to its nonspecific symptomatology, rarity, and lack of

Corresponding Author: Matthew E. Spector, M.D., 1500 E Medical Center Drive, 1904 Taubman Health Center, Ann Arbor, Michigan 48109-5312, Phone: (734) 936-3172, Fax: (734) 936-9625, mspector@ med.umich.edu.

Suggested reviewers: Shamir Chandarana, M.D. (schandar@ucalgary.ca), Michael Brandt, M.D. (dr.m.brandt@gmail.com), Kevin

Fung, M.D. (kevin.fung@lhsc.on.ca) 
literature describing the disease. This case highlights presenting signs and symptoms, imaging, pathology and workup of this disease.

\section{Case Report}

A 50 year-old man presented in clinic with a two-year history of slowly worsening mild hoarseness and dyspnea on exertion. He was a former smoker, with past medical history significant for type 2 diabetes mellitus and obstructive sleep apnea. On examination, he had mild biphasic stridor. Flexible laryngoscopic and bronchoscopic examination in clinic revealed a submucosal multifocal subglottic and tracheal mass with a maximal stenotic area of approximately $60 \%$ narrowing at the $5^{\text {th }}$ tracheal ring. A CT scan of his neck revealed an irregular soft tissue mass measuring $2.8 \times 2.0 \times 2.9 \mathrm{~cm}$ extending from the left lateral and anterior tracheal walls, with involvement of the tracheal cartilage, extending from vertebral level C6 to T2-3 (Figure 1).

The patient was taken to the operating room for microdirect laryngoscopy, bronchoscopy, esophagoscopy and biopsy. He was intubated without difficulty via an awake fiberoptic intubation. Intraoperatively, he was noted to have a diffuse subglottic and tracheal mass leading to significant tracheal stenosis (Figure 2). Multiple biopsies were taken for frozen section. Initial frozen section pathology was consistent with tracheobronchial amyloidosis. After diagnosis, cupped forceps were used to remove portions of the mass anteriorly to improve airway diameter at the maximal stenotic area. The patient's tracheal lumen improved to an approximately $20 \%$ stenosis. The patient was awakened and extubated without difficulty. He had significant improvement in dyspnea postoperatively. Final pathology revealed amorphous eosinophilic deposits within the submucosal tissue and associated with a sparse lymphoplasmacytic infiltrate. The deposits surrounded minor salivary glands and small blood vessels. A Congo red stain highlighted the amorphous deposits which demonstrated apple-green birefringence using polarized light microscopy (Figure 3), consistent with amyloidosis.

On his two month follow-up appointment, patient had overall improvement in dyspnea and hoarseness. He did have some residual voice changes as well as dyspnea with exertion. His pulmonary function tests were within normal limits. He is currently being monitored regularly with plans for repeat debridement versus balloon dilation when he develops recurrent symptoms.

\section{Discussion}

Amyloidosis is an uncommon disease characterized by deposition of protein precursors in various tissues throughout the body, most commonly the heart, liver and kidneys. Amyloidosis can occur in isolated organs as well. Tracheobronchial amyloidosis (TBA) is a rare finding, with only a few hundred cases ever reported.

Amyloidosis originates from deposition of protein subunits in extracellular tissues. The etiology of this disease may incorporate genetic polymorphisms, mutations and local environmental changes, which contribute to altered protein folding, leading to increased 
beta-sheet conformations, and tissue deposition.[1] Light chain amyloidosis (AL) is the most prevalent form of systemic amyloidosis, as well as for localized TBA.[2]

Isolated tracheobronchial amyloidosis has a variable presentation, with common symptoms including hoarseness, stridor, dyspnea, cough, hemoptysis and dysphagia. It is more commonly found in middle-aged males, with a 2:1 predilection versus females.[3] No correlation has been made with tobacco use, with studies reporting less than $50 \%$ of patients with a smoking history.[4] Amyloidosis can present throughout the airway; however, the larynx, trachea, and main bronchi remain the most common sites.[5] Radiographic findings include tracheal wall thickening with calcifications and narrowing of the tracheobronchial airway.[2,6] Although TBA is usually an isolated primary amyloidosis, cases have been reported demonstrating systemic involvement.[4,7]

Screening for symptoms of systemic amyloidosis should be performed in any patient with confirmed TBA. Studies include electrocardiogram, echocardiogram, serum and urine protein electrophoresis, and creatinine level. Evaluation for multiple myeloma should be included in the workup given the frequency of AL amyloidosis, and correlation with myeloma. Cardiac, renal and hepatic disease can range from asymptomatic to end organ failure. Our patient was evaluated by Hematology/Oncology, and work-up showed no evidence of systemic amyloidosis.

Diagnosis is made by tissue biopsy. With isolated TBA, biopsy of lesions within the tracheobronchial airway is necessary for diagnosis. Histologic findings characteristic of amyloidosis include the classic "apple-green" birefringence with Congo red staining on polarized microscopy (Figure 3). Immunohistochemical staining and mass spectrometry can be used to further identify the specific deposited protein subunits.

Treatment of TBA remains a topic under investigation, with mixed results. Bronchoscopic recanalization with ND:YAG and CO2 laser, and mechanical resection have been documented to be successful in individual cases.[3,5] There are reports of successful use of stents in recurrent stenotic tracheobronchial segments.[8] Care should be taken during resection attempts, however, as there has been documented fatal hemorrhage with resection of friable endotracheal and endobronchial amyloid tissue.[9] Advanced local disease may require tracheotomy or laryngectomy to provide a safe airway.[2,5] Treatment with colchicine and systemic glucocorticoids, which have benefit in systemic amyloidosis, have not yielded strong responses in localized TBA. Case reports have documented successful response of TBA to local radiation.[10]

Prognosis of patients with TBA is variable. No systematic reviews have been performed, and most of the literature consists of case reports or small case series. One study documented a 30\% mortality rate from disease after 7-12 years, secondary to progressive obstructive disease[2] while another estimated approximately 30-50\% 5-year survival.[3] Patients with systemic disease often have a much poorer prognosis. Surveillance with close observation for any new pulmonary symptoms, bronchoscopy, pulmonary function tests, and chest CTs can help with predicting disease progression and need for further intervention. 
Conclusion

In summary, TBA is a rare disease with nonspecific pulmonary and upper airway symptomatology. Diagnosis is dependent on tissue biopsy, with Congo red stain demonstrating "apple-green" birefringence. Endoscopic laser or mechanical resection of stenosed tracheal regions has been successfully performed with some success. Prognosis is variable, however, and few studies exist to determine long-term outcomes. Tracheobronchial amyloidosis should remain in an otolaryngologist's differential diagnosis for any patient who presents with a tracheobronchial mass or airway narrowing.

\section{Acknowledgments}

Grant funding: None

\section{References}

1. Bellotti V, Nuvolone M, Giorgetti S, Obici L, Palladini G, Russo P, Lavatelli F, Perfetti V, Merlini G. The workings of the amyloid diseases. Ann Med. 2007; 39:200-207. [PubMed: 17457717]

2. O'Regan A, Fenlon HM, Deamis JF Jr, Steele MP, Skinner M, Berk JL. Tracheobronchial amyloidosis. The Boston University experience from1984 to 1999. Medicine (Baltimore). 2000; 79(2):69-79. [PubMed: 10771705]

3. Ding L, Li W, Wang K, Chen Y, Xu H, Wang H, Shen H. Primary tracheobronchial amyloidosis in China: analysis of 64 cases and a review of the literature. J huazhong Univ Sci Technolog Med Sci. 2010; 30(5):599-603. [PubMed: 21063841]

4. Capizzi SA, Betancourt E, Prakash UB. Tracheobronchial amyloidosis. Mayo Clin Proc. 2000; 75(11):1148-1152. [PubMed: 11075744]

5. Piazza C, Cavaliere S, Foccoli P, TOninelli C, Bolzoni A, Peretti G. Endoscopic management of laryngo-tracheobronchial amyloidosis: a series of 32 patients. Eur Arch Otorhinolaryngol. 2003; 260(7):349-354. [PubMed: 12937908]

6. Yamamoto T, Masayuki M, Mizuno S, Saga T, Itoh H. Primary diffuse tracheobronchial amyloidosis: Radiologic findings. J Thor Imaging. 2001; 16(3):177-180.

7. Crestani B, Monnier A, Kambouchner M, Battesti JP, Reynaud P, Valeyre D. Tracheobronchial amyloidosis with hilar lymphadenopathy associated with a serum monocloncal immunoglobulin. Eur Respir J. 1993; 6(10):1569-1571. [PubMed: 8112453]

8. Alloubi I, Thumerel M, Begueret H, Baste JM, Velly JF, Jougon J. Outcomes after bronchoscopic procedures for primary tracheobronchial amyloidosis: retrospective study of 6 cases. Pulm Med. 2012; 2012:352719. [PubMed: 23326661]

9. Shaheen NA, Salman SD, Nassar VH. Fatal bronchopulmonary hemorrhage due to unrecognized amyloidosis. Arch Otolaryngol. 1975; 101(4):259-261. [PubMed: 1120018]

10. Kurrus JA, Hayes JK, Hoidal JR, Menendez MM, Elstad MR. Radiation therapy for tracheobronchial amyloidosis. Chest. 1998; 114(5):1489-1492. [PubMed: 9824038] 


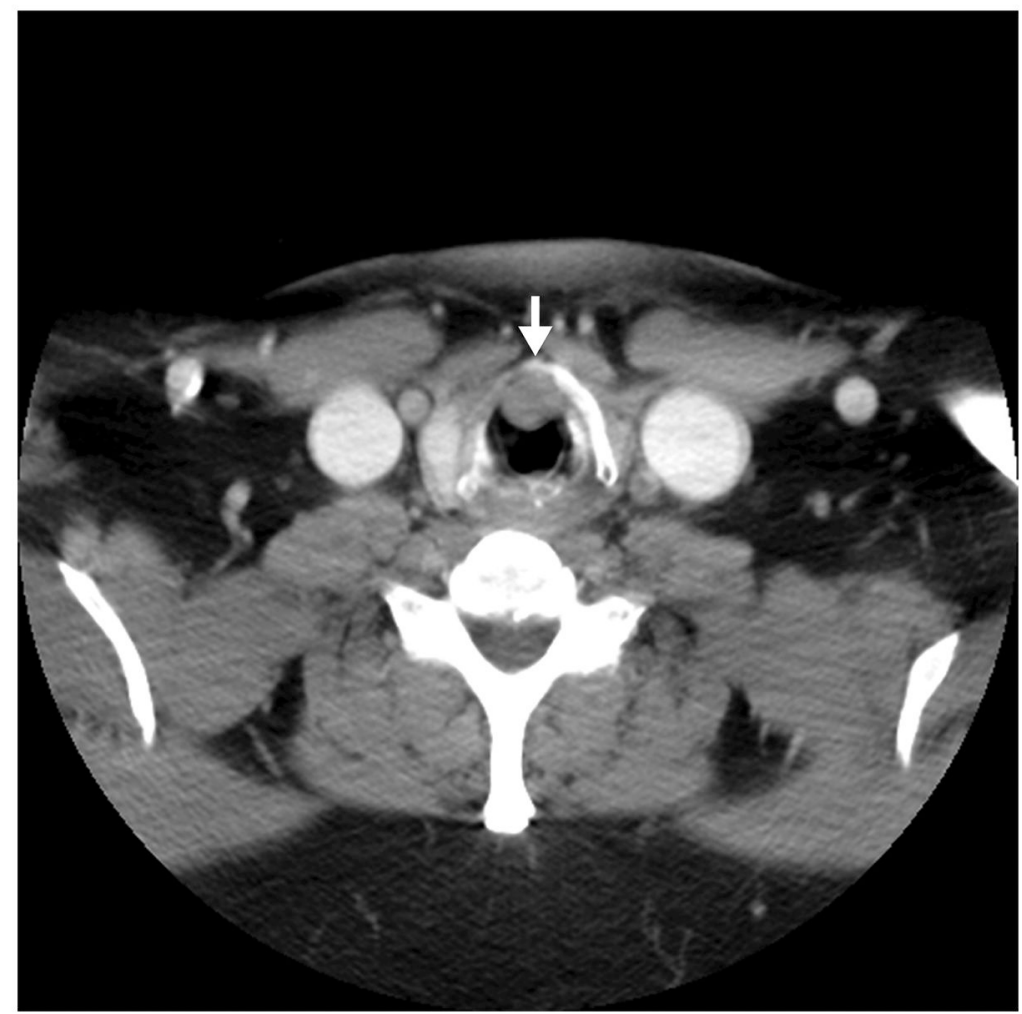

Figure 1.

CT of the neck reveals a soft tissue mass measuring $2.8 \times 2.0 \times 2.9$ along the left lateral and anterior tracheal walls from C6 to T2-3 (white arrow). 


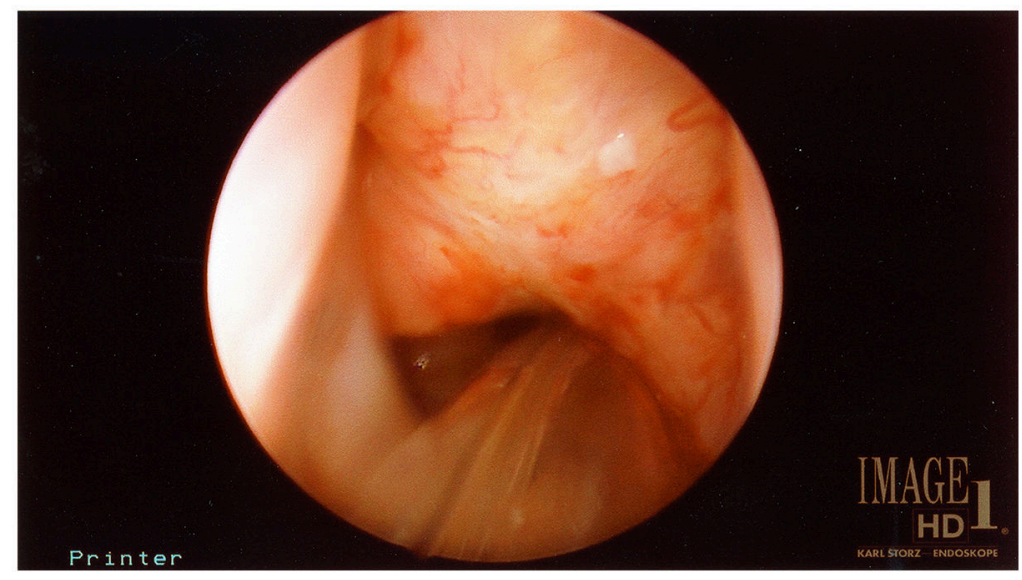

Figure 2.

Intraoperative photo demonstrates the tracheal lesion along the left lateral and anterior tracheal walls. 


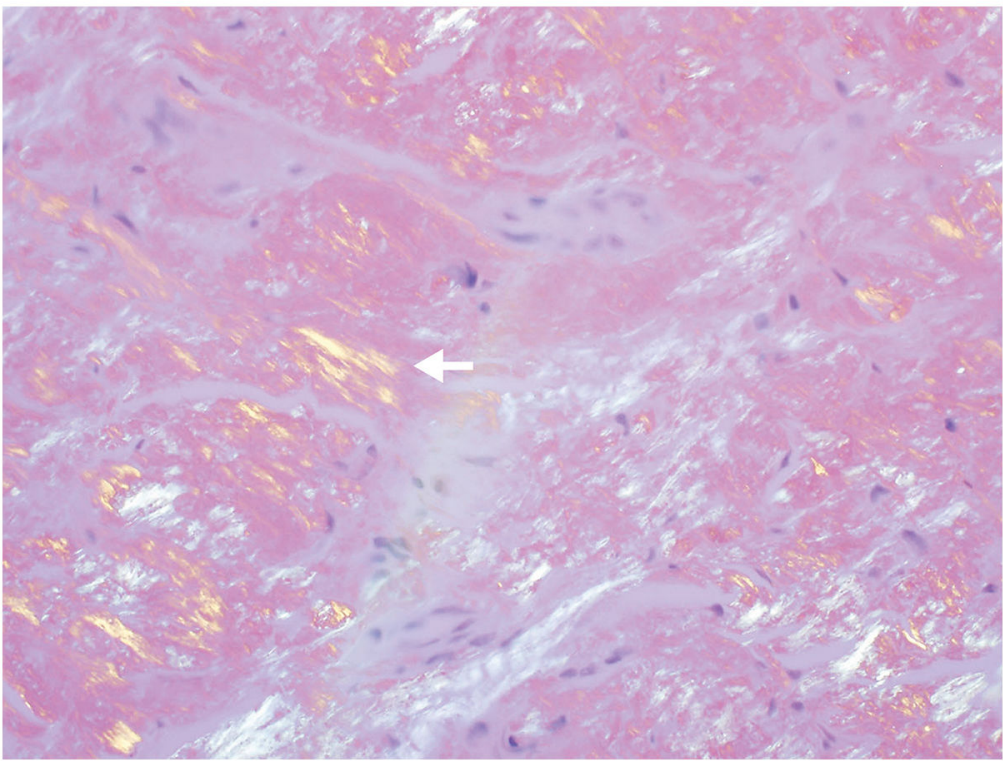

Figure 3.

Congo red stain of tissue demonstrates classic findings consistent with amyloidosis (white arrow). 